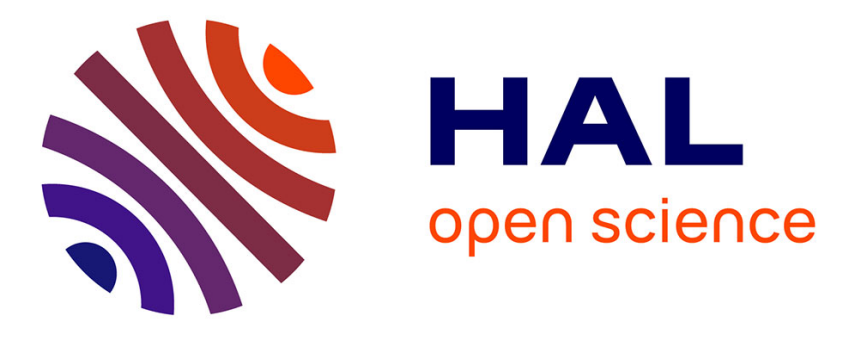

\title{
Reversible 26S Proteasome Disassembly upon Mitochondrial Stress
}

Nurit Livnat-Levanon, Eva Kevei, Oded Kleifeld, Daria Krutauz, Alexandra Segref, Teresa Rinaldi, Zoi Erpapazoglou, Mickael Cohen, Noa Reis, Thorsten Hoppe, et al.

\section{To cite this version:}

Nurit Livnat-Levanon, Eva Kevei, Oded Kleifeld, Daria Krutauz, Alexandra Segref, et al.. Reversible 26S Proteasome Disassembly upon Mitochondrial Stress. Cell Reports, 2014, 7 (5), pp.1371-1380. 10.1016/j.celrep.2014.04.030 . hal-01311781

\section{HAL Id: hal-01311781 https://hal.sorbonne-universite.fr/hal-01311781}

Submitted on 4 May 2016

HAL is a multi-disciplinary open access archive for the deposit and dissemination of scientific research documents, whether they are published or not. The documents may come from teaching and research institutions in France or abroad, or from public or private research centers.
L'archive ouverte pluridisciplinaire HAL, est destinée au dépôt et à la diffusion de documents scientifiques de niveau recherche, publiés ou non, émanant des établissements d'enseignement et de recherche français ou étrangers, des laboratoires publics ou privés. 


\title{
Reversible 26S Proteasome Disassembly upon Mitochondrial Stress
}

\author{
Nurit Livnat-Levanon, ${ }^{1}$ Éva Kevei, ${ }^{2}$ Oded Kleifeld, ${ }^{1,3}$ Daria Krutauz, ${ }^{1}$ Alexandra Segref, ${ }^{2}$ Teresa Rinaldi, ${ }^{4}$ \\ Zoi Erpapazoglou, ${ }^{5}$ Mickael Cohen, ${ }^{5}$ Noa Reis, ${ }^{1}$ Thorsten Hoppe, ${ }^{2}$ and Michael H. Glickman ${ }^{1, *}$ \\ ${ }^{1}$ Department of Biology, Technion-Israel Institute of Technology, Haifa 32000, Israel \\ 2Institute for Genetics, University of Cologne, Cologne 50674, Germany \\ 3Department of Biochemistry and Molecular Biology, Faculty of Health Sciences, Monash University, Melbourne, VIC 3800, Australia \\ ${ }^{4}$ Department of Biology and Biotechnology, University of Rome "La Sapienza," Rome 00185, Italy \\ ${ }^{5}$ Centre National de la Recherche Scientifique, UMR8226, IBPC, and Sorbonne Universités, UPMC, UMR8226, LBMCE, 75005 Paris, France \\ ${ }^{*}$ Correspondence: glickman@tx.technion.ac.il \\ http://dx.doi.org/10.1016/j.celrep.2014.04.030 \\ This is an open access article under the CC BY-NC-ND license (http://creativecommons.org/licenses/by-nc-nd/3.0/).
}

\section{SUMMARY}

In eukaryotic cells, proteasomes exist primarily as $26 S$ holoenzymes, the most efficient configuration for ubiquitinated protein degradation. Here, we show that acute oxidative stress caused by environmental insults or mitochondrial defects results in rapid disassembly of $26 \mathrm{~S}$ proteasomes into intact $20 S$ core and $19 S$ regulatory particles. Consequently, polyubiquitinated substrates accumulate, mitochondrial networks fragment, and cellular reactive oxygen species (ROS) levels increase. Oxidation of cysteine residues is sufficient to induce proteasome disassembly, and spontaneous reassembly from existing components is observed both in vivo and in vitro upon reduction. Ubiquitin-dependent substrate turnover also resumes after treatment with antioxidants. Reversible attenuation of $26 \mathrm{~S}$ proteasome activity induced by acute mitochondrial or oxidative stress may be a short-term response distinct from adaptation to long-term ROS exposure or changes during aging.

\section{INTRODUCTION}

Stress conditions such as UV damage, heat-induced denaturation, peptide-bond cleavage, protein crosslinking, and oxidation of amino-acid side chains can alter protein tertiary structure. Aggregation-prone misfolded proteins, such as those with oxidative damage, require rapid removal, increasing the burden on quality-control and disposal machineries. Reactive oxygen species (ROS) that arise from both environmental and internal sources, such as mitochondrial metabolism, determine the cumulative oxidative stress on cells (Ambrosio et al., 1993). Mitochondrial failure accompanied by ROS production therefore correlates with oxidized protein accumulation and age-related pathologies (de Moura et al., 2010; Friguet et al., 2008; Page et al., 2010; Vendelbo and Nair, 2011). Indeed, damaged mito- chondria correlate with muscle atrophy, neurodegenerative pathologies, and aging itself (Sheridan and Martin, 2010).

Numerous cellular mechanisms are linked to mitochondrial quality control. The ubiquitin-proteasome system (UPS) is the main eukaryotic apparatus for removing aberrant proteins that may contribute to misregulation of processes and disease pathogenesis (Finley, 2009; Glickman and Ciechanover, 2002; Layfield et al., 2001). Fragmented, malfunctioning, or damaged mitochondria can be removed by mitophagy (Green et al., 2011; Rambold et al., 2011), and degradation of specific mitochondrial outer membrane (MOM) proteins by the UPS can promote proper mitochondrial function and morphology (Livnat-Levanon and Glickman, 2011; Taylor and Rutter, 2011). A UPS-driven, mitochondria-associated degradation (MAD) pathway, like the endoplasmic-reticulum-associated degradation [ERAD] pathway), has been postulated (Cohen et al., 2008; Heo et al., 2010).

A UPS-dependent proteolytic event commences with polyubiquitination of a target, followed by recognition of the conjugate by the $26 \mathrm{~S}$ proteasome. Proteasomes unfold ubiquitinated substrates, remove ubiquitin (Ub) tags, and hydrolyze the target into short peptides (Finley, 2009). Proteasomes are highly conserved 2.5 MDa molecular machines consisting of a barrellike $20 \mathrm{~S}$ proteolytic core particle (CP) and a 195 regulatory particle (RP) that recognize and translocate substrates into the 20S CP. Whether one 19S RP or two attach to a single 20S CP defines the two forms of the $26 \mathrm{~S}$ proteasome: $\mathrm{RP}_{1} \mathrm{CP}$ and $\mathrm{RP}_{2} \mathrm{CP}$, respectively (Beck et al., 2012; Glickman et al., 1998b). Access to 20S CP active sites is restricted by a narrow gated channel that is opened upon 19S RP attachment. Only a limited repertoire of misfolded or damaged polypeptides can be degraded by unaided $20 \mathrm{~S} \mathrm{CP}$, yet this may explain the removal of damaged proteins by $20 \mathrm{~S}$ CP during oxidative stress (Bader and Grune, 2006; Davies, 2001). Controlled degradation of globular or structurally stable substrates requires a regulator/ activator complex that traps, unfolds, and feeds substrates into the 20S CP chamber. In cells, the majority of proteasomes exist as 265 holoenzymes, the most efficient configuration for ubiquitinated protein degradation (Bajorek et al., 2003; Kruegel et al., 2011). However, in response to certain stress conditions or upon exposure to oxidants, decreased $26 \mathrm{~S}$ holoenzyme levels 
and increased free $20 \mathrm{~S}$ CP have been reported (Demasi et al., 2003; Reinheckel et al., 1998; Silva et al., 2012; Wang et al., 2010).

To minimize the damaging effects of ROS, aerobic organisms evolved different antioxidant defenses, including the expression of catalases, peroxidases, superoxide dismutases (SODs), and glutathione S-transferases (GSTs) (Hayes et al., 2005; Scandalios, 2005). Ubiquitin and other UPS components are also upregulated upon heat shock or other stresses that induce protein damage (Layfield et al., 2001; Tonoki et al., 2009). Yet, as shown here, 26S proteasome holoenzymes are sensitive to the oxidative conditions under which they disassemble, leading to transient attenuation of ubiquitin-dependent proteolysis. We expand on previous work that described oxidation-driven $26 \mathrm{~S}$ proteasome dissociation (Wang et al., 2010) by demonstrating comparable shifts in proteasome populations from predominantly $26 \mathrm{~S}$ holoenzymes to free $20 \mathrm{~S} \mathrm{CP}$ in response to mitochondrial dysfunction or oxidative stress. We find that proteasome disassembly is reversible both in cells and in vitro. Proteolytically active 20 S CPs and stable 195 regulatory complexes can rapidly reassociate, leading to resumption of ubiquitin-dependent protein turnover in cells, indicating that proteasomes adapt to environmental conditions and cellular needs.

\section{RESULTS}

Proteasomes Dissociate in Response to Oxidative Stress Caused by Mitochondrial Dysfunction

First, we explored whether proteasomes dissociate in response to stimulation of ROS formation due to postdiauxic shift in a respiration-deficient mitochondrial mutant $(\Delta f z 01)$ of yeast. Respiration attempts by defective mitochondria induced ROS production in this strain (Figure S1) and proteasome disassembly was accelerated (Figure 1A). This is a remarkable example of a mitochondrial mutant with a proteasome phenotype. A similar phenomenon was observed in a strain in which a proteasome subunit-encoding gene was mutated (rpn11-m1; Figure 1A). Fragmented mitochondria networks were previously reported in rpn11-m1 (Rinaldi et al., 2002). Extending those observations, we measured high levels of intracellular ROS (versus wild-type [WT]) in both strains ( $\Delta$ fzo1 and rpn11-m1; Figure S1). Cellular damage due to defective mitochondria attempting to respire on low concentrations of nonfermentable carbon may be the root cause of the observed altered proteasome activity (Figure 1).

Next, we explored whether malfunctioning mitochondria can directly induce proteasome disassembly, by testing the effect of a mitochondrial respiration inhibitor (Antimycin A, a Cytochrome C reductase inhibitor; Chen et al., 2003; Turrens, 1997) on proteasomes. We found that treating rapidly dividing yeast cells with Antimycin A resulted in rapid disassembly of $26 \mathrm{~S}$ proteasomes in vivo (Figure 1B), but had no direct effect on purified proteasomes (Figure 1B). Proteasome disassembly in cells correlated with altered oxidative phosphorylation in mitochondria (i.e., an inability to utilize nonfermentative carbon; Figure 1C), ROS release (Chen et al., 2003; Lin and Beal, 2006; Sedensky and Morgan, 2006), and pervasive protein oxidation (Figure 1C). Attenuated proteasomes were also observed in cell cultures subjected to direct oxidation. In both yeast and mammalian cells, 26S proteasome activity decreased with exposure to increasing concentrations of external oxidizing reagents (Figure 1D). Concomitantly with diminished $26 \mathrm{~S}$ proteasome activity, increased 20S CP activity was measured, suggesting that proteasome active sites were not hampered, but rather the proteasome population shifted from predominantly 265 holoenzymes to primarily free 20 S CP. 20 S CP was detached in its latent form and was chemically activatable (by miniscule concentrations of SDS; Glickman et al., 1998a). Notably, even at these considerably high concentrations of $\mathrm{H}_{2} \mathrm{O}_{2}$ in the medium, cells were viable for at least a couple of hours, and growth resumed (together with reassociated proteasomes) when the oxidants were removed from the media or the media were neutralized with a reducing agent (Figures 1D, middle panel, and $2 \mathrm{~F}$ ). Thus, our findings demonstrate that proteasome conformation is sensitive to oxidative stress, and despite the essential role of proteasomes in protein turnover, under the tested conditions, cells maintain viability even though the activity of the $26 \mathrm{~S}$ holoenzyme is attenuated.

\section{Proteasomes Exhibit Reversible Redox-Dependent Dissociation/Reassociation}

We evaluated the direct effect of oxidation on the proteasome by exposing purified proteasomes to oxidants and resolving the breakdown products by nondenaturing gels (in order to keep complexes intact and proteolytically active for enzymatic or biochemical analysis). A dose-dependent detachment of active 20S CP from 26S proteasomes was observed upon addition of $\mathrm{H}_{2} \mathrm{O}_{2}$ (Figure 2A, left panel), and the migration pattern mimicked the behavior of proteasomes in stressed cells (Figure 1). In parallel to increased levels of the 20S CP form, a second proteolytically inactive complex containing 19S RP subunits appeared at the expense of the diminishing $26 \mathrm{~S}$ (Figure 2A, middle and right panels). In yeast cells too, simultaneous accumulation of complexes containing 19S RP or 20S CP subunits was observed in response to acute oxidative stress concomitantly with diminished $26 \mathrm{~S}$ proteasome levels (Figure $2 \mathrm{~A}$, right). We determined the makeup of these complexes by mass spectrometry (MS)based, label-free quantitative analysis. All 19S RP subunits in the proteolytically inactive complex were identified at roughly stoichiometric levels (Figure 2B; Table S1). The quantity of intact regulatory $19 S$ complexes in the treated samples relative to the untreated control increased significantly. Released 20S particles were active but latent, and could be reactivated by treatment with a low level of SDS (Figure 2A), similar to 20S CP generated in stressed cells (Figure 1). Thus, these findings demonstrate that in response to oxidative stress, 26S proteasome holoenzymes dissociate into two subcomplexes, which remain stable and intact.

We investigated whether proteasome disassembly is an irreversible consequence of oxidative damage or an environmental-sensing mechanism by testing the effect of a reducing reagent (dithiothreitol [DTT]) on cells after dissociation of proteasome complexes. Proteasome disassembly was partially reversed within minutes of adding DTT to the cell media (Figure 2C). Even disassembly of proteasomes that occurred naturally over time in respiratory-defective cells (rpn11-m1) was 


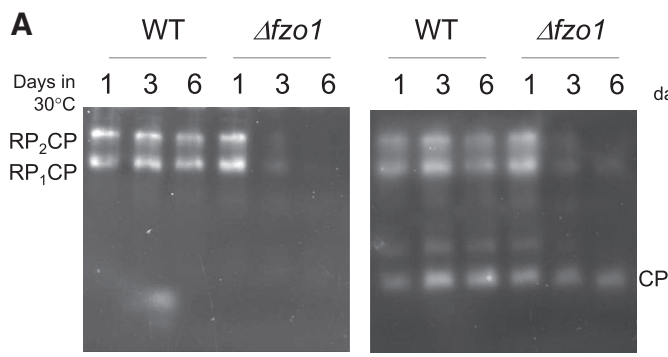

activated

B

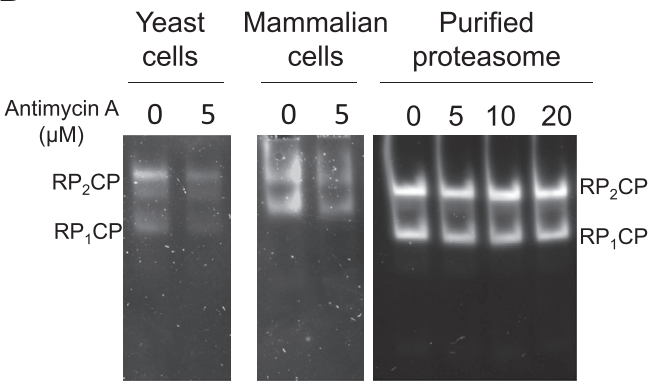

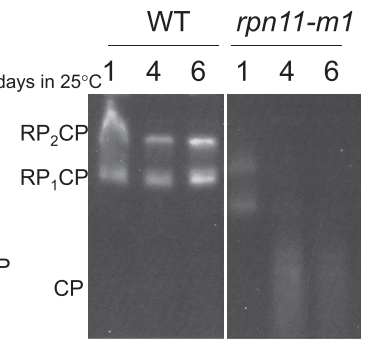

C

$\frac{\mathrm{WT}}{14} \quad 6 \quad \frac{r p n 11-m 1}{14}$

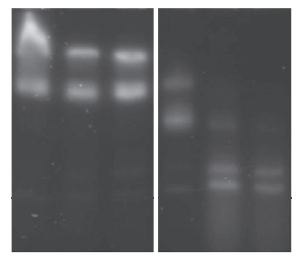

activated

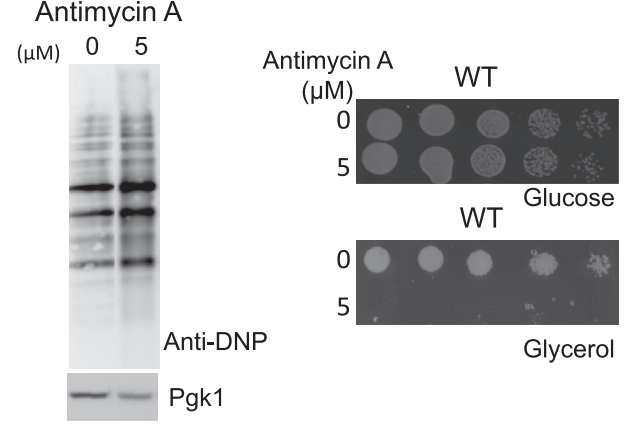

D

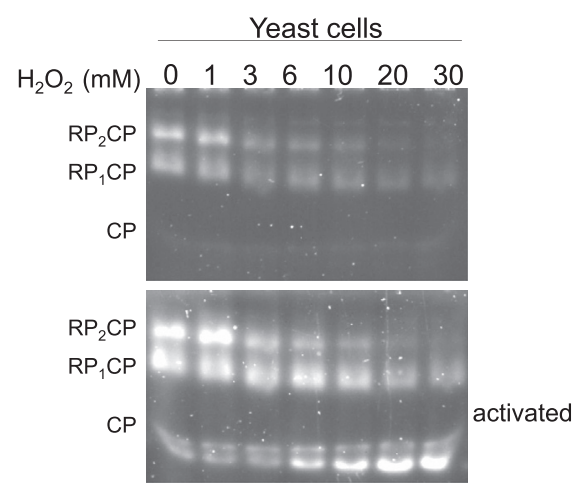

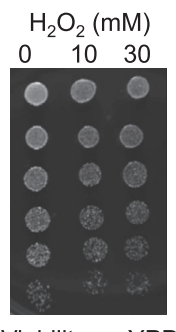

Viability on YPD

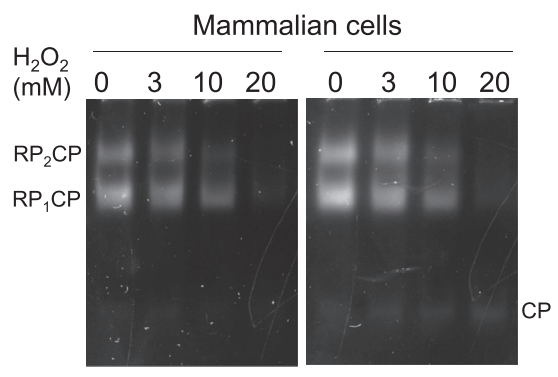

activated

Figure 1. Fragile Proteasomes in Response to Oxidative Stress

(A) Proteasome disassembly following postdiauxic shift. $\Delta f z 01, r p n 11-m 1$, and their isogenic WT strains were shifted from fermentative logarithmic growth to diauxic respiration by glucose starvation. At the indicated days, equal numbers of cells were harvested, lysates were separated by nondenaturing PAGE (native gel), and proteasome activity was detected by in-gel peptidase activity. The main proteasome species are marked $\left(\mathrm{RP}_{2} \mathrm{CP}\right.$, doubly capped $26 \mathrm{~S} ; \mathrm{RP}_{1} \mathrm{CP}$, singly capped 26S; CP, 20S CPs). Latent 20S CP was activated by infusing $0.02 \%$ SDS ("activated").

(B) Proteasome attenuation in response to mitochondria respiration. Yeast (left) or mammalian cells (middle) were incubated with $5 \mu \mathrm{M}$ Antimycin A for $3 \mathrm{hr}$ and proteasome activity was monitored by native gel. Purified proteasomes were tested with Antimycin A for control (right).

(C) Antimycin A promoted protein oxidation. Cellular extracts following Antimycin A treatment were assayed for oxidized proteins by incubation with DNPH, a reagent for oxidized amino-acid side chains (left). Following Antimycin A treatment, cells were also scored as 10-fold dilutions onto glucose-containing (top) or glycerol-containing (bottom) media to evaluate respiration capability.

(D) Direct oxidation led to proteasome dissociation. $\mathrm{H}_{2} \mathrm{O}_{2}$ was added to logarithmically growing WT yeast or mammalian BHK-21 cells for 30 min prior to harvesting and visualization of proteasome by in-gel peptidase assay.

partially corrected by adding DTT (Figure 2D). Diminished $26 \mathrm{~S}$ proteasome levels in cells chemically treated for a few hours with a mitochondria inhibitor were also rapidly restored by adding DTT (Figure 2E). Reassembly of oxidized proteasomes in vivo did not require de novo synthesis of subunits, as it occurred spontaneously even when protein synthesis was blocked with cycloheximide ( $\mathrm{CHX}$, a ribosome inhibitor; Figure 2F). Consistent with these findings, in vitro, purified $26 \mathrm{~S}$ proteasomes were rebuilt upon addition of DTT alone (i.e., without chaperones or other factors) to the buffer (Figure 2G). These findings indicate that reduction-oxidation (redox) of proteasome components directly affects holocomplex stability, and that oxidized subcomplexes remain competent and can reassemble under permissive conditions.

We focused on the role of thiol residues in the dissociation/ reassembly process (which was postulated due to the reversible nature of this redox-driven process) by repeating the experiments with diamide, a cysteine-specific reagent that crosslinks 
A

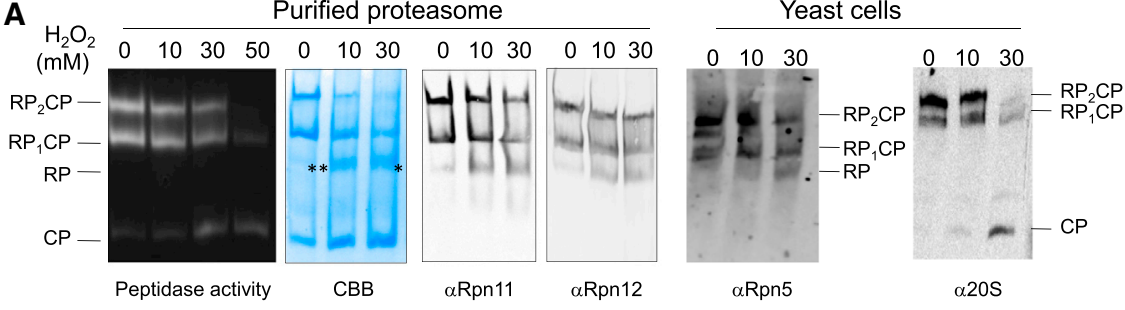

B

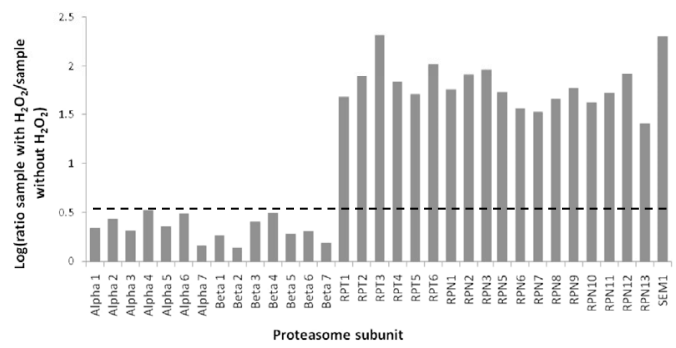

C

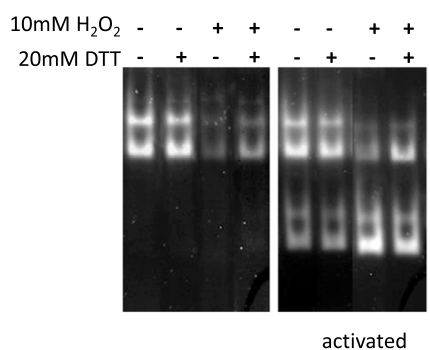

\section{D}

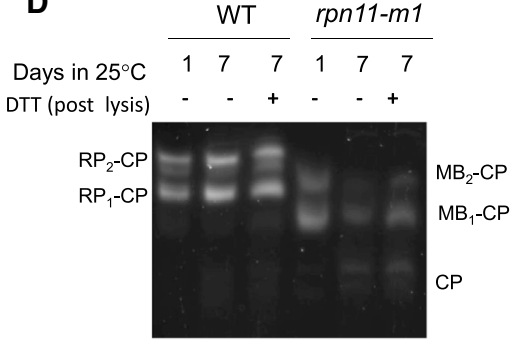

E

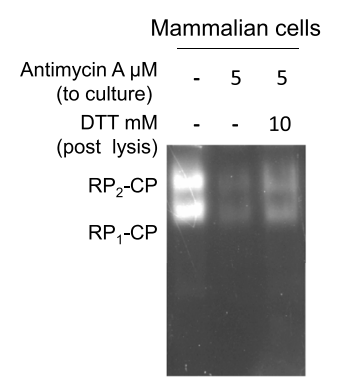

$$
\mathbf{F}
$$

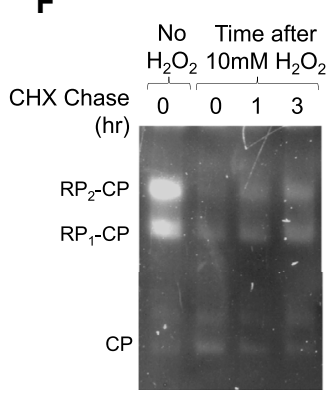

G

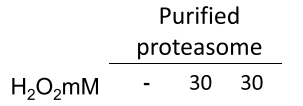

DTT $\mathrm{mM} \quad$ - $\quad$ - 30

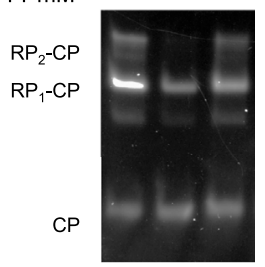

Figure 2. Reversible $26 \mathrm{~S}$ Proteasome Disassociation in Response to Oxidative Stress

(A) Free 20S CP and 19S RP detached from oxidized proteasomes. Purified 26S proteasomes were exposed to increasing concentrations of $\mathrm{H}_{2} \mathrm{O}_{2}$ for $30 \mathrm{~min}$ and visualized by native gel: peptidase activity (left), protein content, and immunoblots for proteasome subunits Rpn11 or Rpn12 (middle). $\mathrm{H}_{2} \mathrm{O}_{2}$-treated yeast cells (as in Figure 1D) were immunoblotted for the 19S RP subunit (Rpn5) or the 20S CP subunit (right).

(B) Intact 19S RP subcomplex dissociated from $26 \mathrm{~S}$ proteasomes. The subunit composition of proteasome species was determined by label-free MS/MS quantification. Proteasome subunits compared before $\left(^{(*}\right)$ and after $\left(^{*}\right) \mathrm{H}_{2} \mathrm{O}_{2}$ treatment verified the presence of inactive 19S RP (Table S1). (C-G) Proteasome disassembly was reversed by a reducing agent.

(C) Logarithmic-phase yeast was treated with $10 \mathrm{mM} \mathrm{H}_{2} \mathrm{O}_{2}$ for 30 min and lysed as is or with $20 \mathrm{mM}$ DTT for in-gel peptidase activity.

(D) WT and rpn11- $m 1$ cells were grown at $25^{\circ} \mathrm{C}$ and lysed with or without $10 \mathrm{mM}$ DTT.

(E) Mammalian BHK-21 cells were treated with Antimycin A and lysed with or without $10 \mathrm{mM}$ DTT for visualization by native gel.

(F) Reassembly of dissociated proteasomes did not require de novo synthesis. WT cells at logarithmic phase (left lane) were treated with $\mathrm{H}_{2} \mathrm{O}_{2}$ for 30 min (second lane). $\mathrm{H}_{2} \mathrm{O}_{2}$ was removed, and cells resumed growth in the presence of $200 \mu \mathrm{g} / \mathrm{ml}$ $\mathrm{CHX}$ to block new protein synthesis. Proteasomes in all samples were compared by native gel.

(G) Purified 26S proteasomes (left) were treated with $30 \mathrm{mM} \mathrm{H}_{2} \mathrm{O}_{2}$ for 30 min, $\mathrm{H}_{2} \mathrm{O}_{2}$ was removed, and the sample was split (half loaded "as is" [middle] and half supplemented with $10 \mathrm{mM}$ DTT [right]).

epitope-tagged N-ethylmaleimide (NEM; Figure $3 \mathrm{~B})$. In this assay, only cysteines that were crosslinked in the free $20 \mathrm{~S}$ and 19S subcomplexes were labeled by biotin-NEM (see details in legend for Figure 3 and in Supplemental Experimental

proximal thiols (Kosower and Kosower, 1995; Manalo et al., 2002). Diamide alone was sufficient to drive dissociation of 26S proteasomes into intact 20S CP and 19S RP species (Figure $3 A$ ), mimicking the experimental results obtained by other means of oxidation (Figures 1 and 2). Adding DTT reversed the diamide effect, thereby demonstrating that redox of thiol groups within the proteasome was sufficient to drive proteasome dissociation/reassembly (Figure 3A). Although crosslinking subunits may stabilize a complex, the $26 \mathrm{~S}$ holocomplex was actually split into two subcomplexes upon formation of internal dicysteine bonds, suggesting that dicysteine formation did not link the two subcomplexes, but rather occurred within each subcomplex. To map the oxidation-prone subunits, we labeled cysteines that were oxidized upon proteasome disassociation with an
Procedures). Evidently, multiple subunits in both 19S RP and $20 S$ CP directly participated in dicysteine bond formation. MS of oxidized and labeled proteasomes identified the participating cysteines in each subunit, and we compared cysteine modifications between diamide-treated and -untreated proteasomes by differentially labeling cysteines in two proteasome preparations (Table S2). Given that oxidized cysteines (a.k.a. cystines) were identified in dissociated 20S CP and 19S RP subcomplexes, but not in $26 \mathrm{~S}$ holoenzymes, we conclude that crosslinks formed within each of the two subcomplexes, but not across the 19S RP-20S CP interface. We propose that such cystines (disulfur bonds) probably altered the surface of the interaction and weakened the 19S RP-20S CP association. The formation of disulfur bonds may even protect proteasomes from more severe, 
A

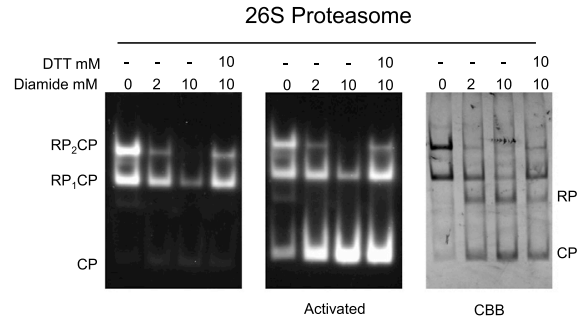

B

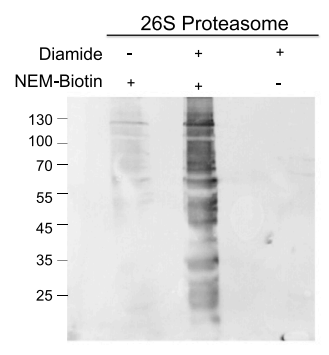

Streptavidin-HRP
C

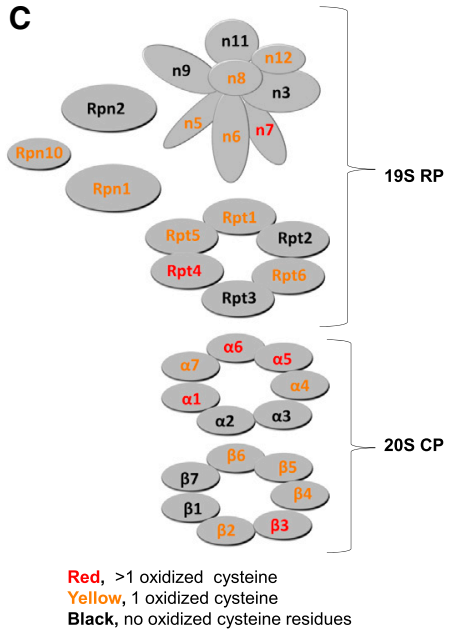

Figure 3. 26 S Dissociation by Dicysteine Crosslinking (A) A specific cysteine modifier, diamide, drives reversible dissociation of $26 \mathrm{~S}$ proteasomes. Purified $26 \mathrm{~S}$ proteasomes were incubated with diamide for $30 \mathrm{~min}$ at $30^{\circ} \mathrm{C}$ and resolved by native gel for activity (left) and protein content (right); $10 \mathrm{mM}$ DTT was added to one sample.

(B) Cysteines were crosslinked in dissociated 20S CP and 19S RP. After treatment with $5 \mathrm{mM}$ diamide, proteasomes were denatured with $6 \mathrm{M}$ urea and all remaining reduced cysteines were irreversibly alkylated by $2 \mathrm{mM}$ iodoacetamide. Next, the crosslinked cysteines were reduced by $8 \mathrm{mM} \mathrm{DTT}$, irreversibly labeled with NEM-biotin, and detected by horseradish peroxidase (HRP)-streptavidin.

(C) Illustration of dicysteine bonds in oxidized 19S and 20 S subcomplexes identified by MS/MS. Red, single oxidized cysteine; yellow, multiple cysteines; gray, no cysteines. Dicysteine bonds were confined to each subcomplex and did not span the 19S-20S interface.

irreversible thiol oxidation (e.g., due to sulfinic or sulfonic acids) in a manner similar to that observed for glutathione, one of the common antioxidants in cytosols of eukaryotes (Hayes et al., 2005; Scandalios, 2005).

Slowdown of Proteasome-Dependent Substrate Turnover in Response to Acute Oxidative Stress Next, we assessed the correlation between oxidative stressinduced decrease in the cellular levels of active 26S proteasomes and substrate turnover. Induction of cellular oxidative stress in live $C$. elegans by knocking down mitochondrial respiration components with RNAi (Figure 4A, left) resulted in accumulation of a ubiquitin-fusion degradation model substrate (UFD; Figure 4A, right). Stabilization of UFD substrates is typical of 265 proteasome dysfunction (Bajorek et al., 2003; Rubin et al., 1998; Segref et al., 2011). Supplying worms with an antioxidant (N-acetylcysteine) restored UFD-substrate degradation (Fig-

ure 4B), even when mitochondria were still genetically impaired (Segref et al., 2014), suggesting that the efficiency of UPS was responsive to cellular oxidative conditions. Notably, the effects of mitochondrial mutations (isp-1, nuo-6, and gas-1) on stabilization of a UFD substrate were remarkably similar to those obtained by direct interference with $26 \mathrm{~S}$ function (rpn-6 and rpn-11). Slower turnover of a natural UPS substrate, Fzo1, was reflected by extended half-life following exposure of yeast to an oxidant (Figure 4C). Accumulation of high-molecular-weight polyubiquitin conjugates in oxidative-stressed cells was a broad phenomenon (Figure 4D; see also Wang et al., 2010) and indicated that bulk protein turnover was generally slowed down. Thus, our findings suggest that UPS activity is malleable and responds to the redox potential of the environment. Notably, proteasome conformation is also modulated by similar conditions, reinforcing the link between the two observations.

\section{Mitochondrial Defects and Antioxidative Stress Response Are Induced by Reduced Proteasome- Dependent Proteolysis}

We evaluated the contribution of proteasome capacity to cellular oxidative stress by monitoring the effect of proteasome inhibition on mitochondrial morphology, oxidation damage, and antioxidative stress response. In most tissues during typical growth conditions, mitochondria form an ever-changing branched network driven by fusion of membrane tips and fission into smaller organelles (Okamoto and Shaw, 2005). Such a tubular-reticular network of mitochondria was visualized in live C. elegans (in the body-wall muscle cells; Figure 5A, left). A dense interlocked network of mitochondria is also apparent in many mammalian cells (see example in Figure 5B; left) and in WT yeast (Altmann and Westermann, 2005; Merz and Westermann, 2009; Rinaldi et al., 2002). Knocking down individual proteasome subunits by RNAi (20S subunits pas- 1 and pbs- 2 or 19 s subunits rpn-6, $r p n-11$, and rpt-1) led to small fragmented mitochondria in C. elegans (Figure 5A). Likewise, within hours of chemical inhibition of proteasomes in mammalian cells, the mitochondrial morphology shifted rapidly from the characteristic tubular network to multiple small and fragmented organelles (Figure 5B). Although in both of these examples proteasome function was significantly decreased, the phenotypic outcome was numerous small, rounded mitochondria. This phenotype is also produced by direct inhibition of oxidative phosphorylation, such as that caused by antimycin A (Sheridan and Martin, 2010). In yeast too, fragmented mitochondria have been characterized in proteasome mutants (Rinaldi et al., 2002). In addition, proteasome mutants accumulated oxidized proteins (Figure 5C), similar to the results observed for mitochondrial malfunction (Figure 1). Thus, these findings suggest that malfunctioning mitochondria appear to be another outcome of insufficient proteasome/UPS capacity.

Fragmented mitochondria (Figures $5 \mathrm{~A}$ and $5 \mathrm{~B}$ ), elevated ROS levels, and accumulation of damaged proteins (Figures S1 and $5 \mathrm{C})$ all trigger the antioxidative stress response. Interestingly, attenuation of UPS by RNAi knockdown of proteasome subunits did so as well, as reflected by induction of the gst-4 gene in C. elegans (Figure S3). GSTs serve as key enzymes in the antioxidative stress response by maintaining appropriate cellular levels 
A Marker for oxidative stress

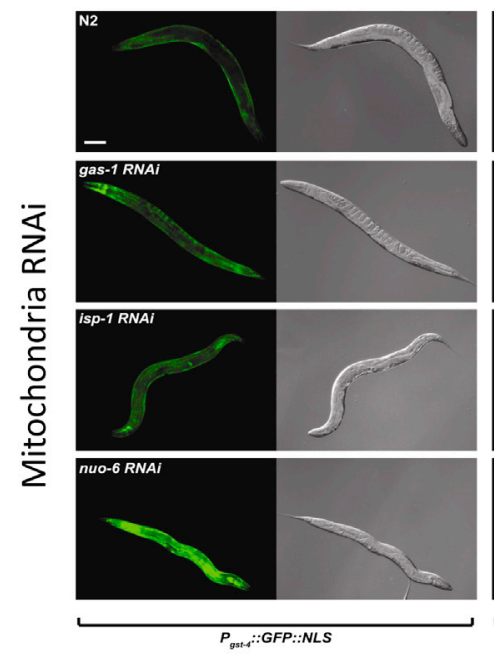

C

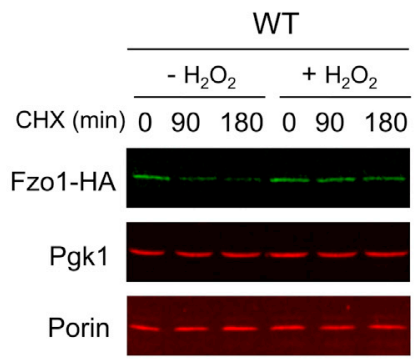

Marker for UPS stress
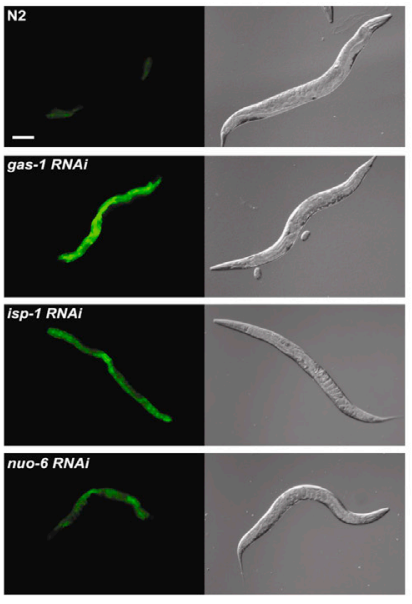

$P_{\text {surs: }: \text { UbiV-GF }}$

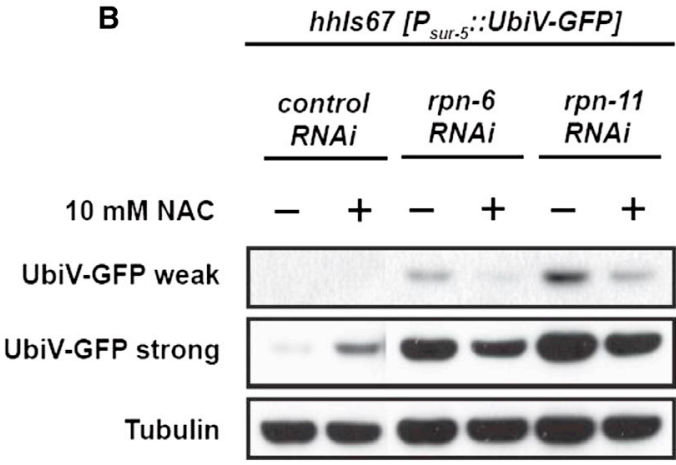

D

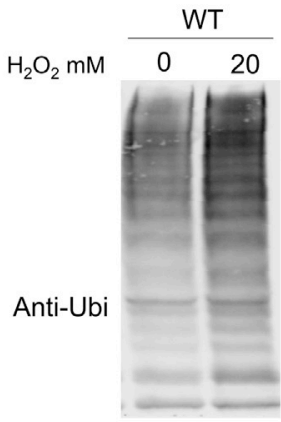

Pgk1

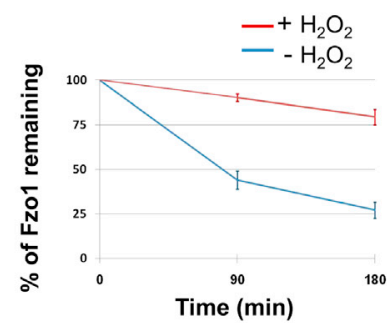

Figure 4. Acute Oxidative Stress Attenuates Ubiquitin-Dependent Proteolysis

(A) Defects in mitochondrial respiration stabilized a UFD substrate. C. elegans expressing an oxidative stress marker, GFP::NLS, under the control of the gst-4 promoter (left), or a model UPS substrate, UbiV-GFP, under the control of the sur-5 promoter (right), were fed with RNAi-expressing bacteria and imaged 72 hr after L1 stage (fluorescent and differential interference contrast [DIC], right). Scale bar indicates $100 \mu \mathrm{m}$.

(B) Accumulation of UbiV-GFP was reversed by reduction. Nematodes prepared as in (A) were exposed to N-acetylcysteine (NAC) for 24 hr or kept without the antioxidant. Total lysates were immunoblotted by anti-GFP and anti-tubulin. Higher exposure showed that NAC had no effect on the UbiV-GFP substrate in WT nematodes (left), but aided turnover when mitochondrial genes or proteasomal genes were knocked down (Figure S3).

(C) A UPS substrate, Fzo1, accumulated upon oxidative stress. Logarithmically growing WT yeast expressing $\mathrm{HA}-\mathrm{Fzo} \mathrm{were}$ exposed to $20 \mathrm{mM} \mathrm{H}_{2} \mathrm{O}_{2}$ for 30 min, supplemented with $200 \mu \mathrm{M} \mathrm{CHX}$ to block protein synthesis, and immunoblotted for residual Fzo1 (top), Pgk1 (middle), and mitochondrial Porin (bottom). Quantification of Fzo1-HA is shown in the graph on the right.

(D) Accumulation of polyubiquitin conjugates after acute oxidative stress. Lysates of log-phase WT yeast exposed to $\mathrm{H}_{2} \mathrm{O}_{2}$ were assayed for ubiquitin conjugates or Pgk1 (normalization control) relative to untreated cells.

of reduced GSH and inactivating oxidized functional groups on cellular macromolecules (Hayes et al., 2005; Scandalios, 2005). Another potent detoxification enzyme, SOD, was similarly elevated in a proteasome mutant (rpn11-m1; Figure 5D), emphasizing the antioxidative stress response as a common consequence of decreased proteasome capacity.

In order to grasp the full extent of reduced proteasome activity on cellular response pathways, we compared the rpn11-m1 proteome in logarithmic phase with that of its congenic WT using stable isotope labeling by amino acids in cell culture (SILAC) followed by a comprehensive MS/MS analysis of total cell lysates (see Table S3). Significant enrichment of proteins related to oxidative stress response, carbohydrate catabolism, or pro- tein-folding pathways was measured in the rpn11-m1 proteome relative to the WT (Figure 5E). Notably, induction of GST genes was observed in response to diminished levels of the same proteasome subunit Rpn11 in yeast (rpn11-m1; Figure 5E) and in C. elegans (rpn-11; Figure S3), reflecting an evolutionarily conserved strategy to combat oxidative stress caused by insufficient proteasome capacity. Constitutive induction of reductases, scavenging enzymes, and chaperones (as in Figure 5F) may explain how some strains with proteasome defects sustain viability despite impaired mitochondria, inefficient oxidative phosphorylation (Figures 5A, 5B, and S2), increased ROS production (Figure S1), and misfolded/damaged proteins (Figure 5C). 
A
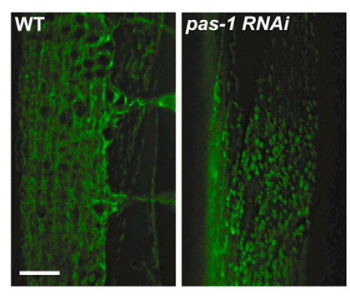
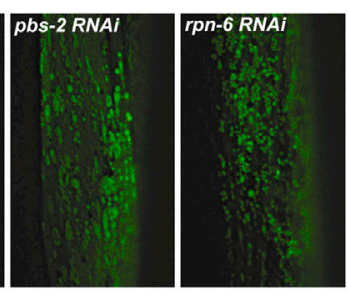

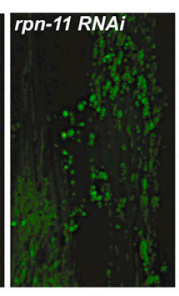

B

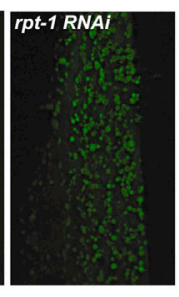

No treatment

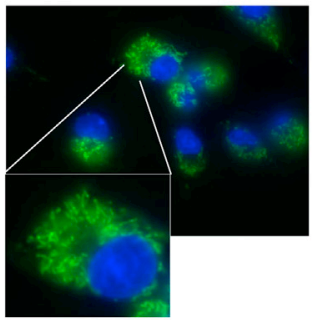

MG132

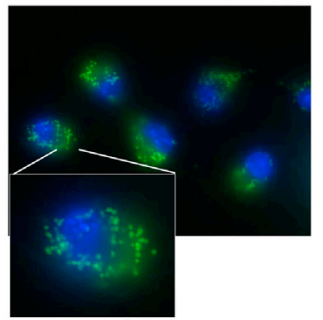

C

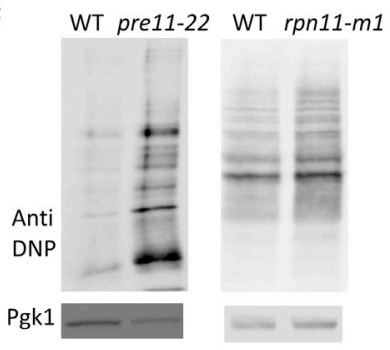

E
D

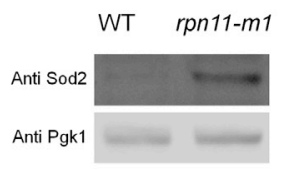

Protein ID

YGR088W

YLL026W

YER103W

YFL014W

YGR209C

YKR076W

YDR453C

YJR104C

YHL021C

YDR353W

YHROOBC

YBLO64C

YJRO77C

YMR308C

YIL034C

YBL003C

YMR246W

YJL208C
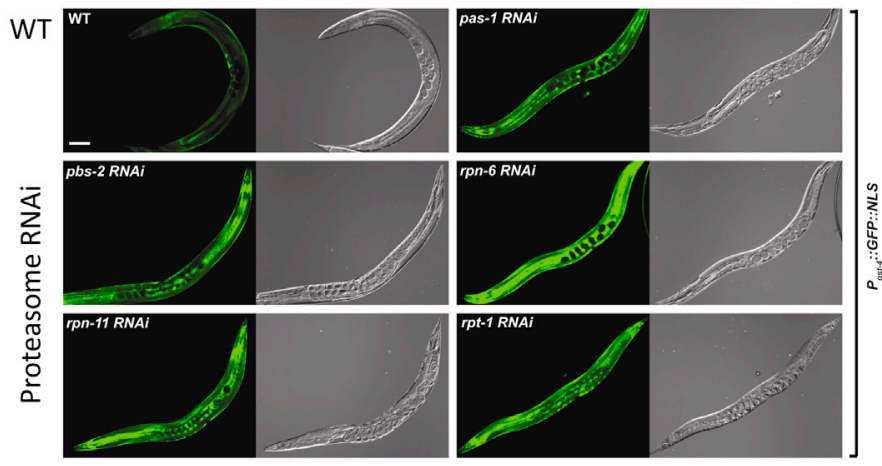

Marker for oxidative stress (GFP::NLS under control of gst-4)

F

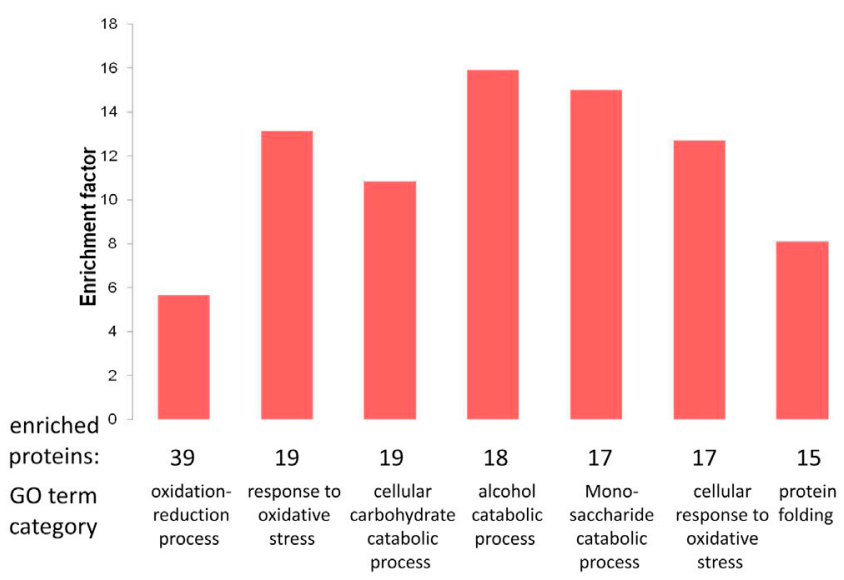

Figure 5. Attenuated Proteasomes and Impaired Mitochondria Display Antioxidative Stress Response Markers

(A) Defective proteasomes cause mitochondrial fragmentation. Fluorescent images of the mitochondrial network in body-wall muscle cells from 2-day-old adult nematodes expressing mitochondria-localized GFP under control of the myo-3 promoter that were fed with RNAi bacteria from L3 larval stage for $72 \mathrm{hr}$. Scale bar indicates $8 \mu \mathrm{m}$

(B) Proteasome inhibition fragments mitochondria in mammalian cells. BHK-21 cells were treated with $10 \mu \mathrm{M}$ MG132 for $3 \mathrm{hr}$, mitochondria were stained with MitoTracker green FM, and nuclei were visualized using Hoechst at $63 \times$ magnification.

(C) Oxidized proteins correlated with impaired UPS. Total cell extracts from proteasome mutants (pre11-22 and rpn11-m1) and WT yeasts were reacted with DNPH to evaluate oxidized proteins. Pgk1 immunoblot served for normalization.

(D) Proteasome defects induce the oxidative stress response. Nematodes expressing GFP::NLS under the control of the gst-4 promoter were fed with RNAiexpressing bacteria from L3 larval stage for $48 \mathrm{hr}$ (PAS-1 and PBS-2 are $20 \mathrm{~S} \alpha 1$ and $\beta 2$ subunits, respectively) and imaged (left: GFP fluorescent; right: DIC). WT and rpn11-1 strains were immunoblotted to detect SOD levels in whole-cell extract.

(E) Upregulation of the antioxidative stress response in a proteasome mutant, rpn11-1. WT and rpn11-m1 proteomes were compared by SILAC MS/MS analysis of normalized cell lysates. Intensity ratios of identical peptides (heavy-to-light) were obtained by running the entire combined peptide data set through MaxQuant software (Cox and Mann, 2008). More than 100 proteins were significantly enriched in the proteome of rpn11-m1 relative to WT (Table S3). Representative proteins enriched in rpn11-m1 (red), unchanged (yellow), or decreased in rpn11-m1 (green) are listed.

(F) Biological pathways significantly enriched in rpn11-m1 cells (rpn11-m1/WT > 3) were classified using the AmiGo program (right). For each category, the "enrichment factor" is the ratio between the category in highly enriched proteins relative to its proportion of the total proteome (red bar), along with the number of proteins in the enriched sample. 


\section{DISCUSSION}

Prolonged proteasome inhibition often causes cell death. A decrease in proteolytic capacity may lead to pervasive defects such as protein aggregation, neurodegeneration, certain cancers, aging, and even apoptosis. It was surprising that despite temporary 26S proteasome disassembly and attenuation of ubiquitin-conjugate turnover that followed acute short-term oxidative stress, organisms/cells remained viable. The reversible nature of proteasome disassembly suggests an adaptive response rather than protein damage. "Pausing" the ongoing turnover of ubiquitinated proteins may allow cells to redirect resources elsewhere. Simultaneously, thiol groups on 265 proteasome subunits are blocked (by dicysteine formation or glutathionylation) and protected from irreversible oxidation or damage. For instance, glutathionylation of cysteine residues on the surface of $\alpha$-subunits was found to open the $20 \mathrm{~S}$ channel, thereby activating proteolysis (Silva et al., 2012). In this manner, cysteine modifications may influence ratio of $20 \mathrm{~S}$ to $26 \mathrm{~S}$, as well as their relative activities. Subcomplexes released under stress upon $26 \mathrm{~S}$ proteasome oxidation may also play new roles. The proteolytic activity of free $20 \mathrm{~S} \mathrm{CP}$ is expected to be less selective than that of $26 \mathrm{~S}$ holoenzymes and thus more efficient in tackling misfolded/damaged proteins (Tsvetkov et al., 2012). Indeed, free $20 \mathrm{~S}$ CP is highly efficient in proteolyzing certain loosely folded or unstructured proteins in vitro (Bajorek et al., 2003; Glickman et al., 1998a) and in a ubiquitin-independent, 20S-dependent manner in vivo (Davies, 2001; Mukhopadhyay and Riezman, 2007; Orlowski and Wilk, 2003; Shringarpure et al., 2001, 2003). Thus, CPs may aid the oxidative stress response by degrading oxidized and damaged proteins (Bader and Grune, 2006; Grune et al., 2003; Reinheckel et al., 1998; Sitte et al., 1998). Shifting the proteasome population from a majority of $26 \mathrm{~S}$ to a greater proportion of $20 \mathrm{~S}$ CP may be a mechanism for adapting to the proteolytic capacity.

Proteostasis may be preserved by inducing compensatory mechanisms even if the UPS is slowed down. Induction of antioxidative stress response, detoxification enzymes, chaperones, heat shock proteins, and mitophagy may alleviate the flux through the UPS, whereas directing ubiquitin molecules toward other protein quality-control systems, such as autophagy and trafficking, may take up some of the slack (Yao, 2010; Ziv et al., 2011). For example, administering a nontoxic low dose of the proteasome inhibitor PS-341 (bortezomib) to rats induced elevation of some of the same antioxidative defense enzymes that we identified in rpn11-m1 (glutathione reductase, glutathione synthetase, glutathione peroxidase 2, and SOD2) and increased their ability to overcome oxidative stress (BardagGorce et al., 2011). The mutual dependence of proteostasis pathways such as the UPS, autophagy, heat-shock system, and antioxidative stress response may explain their similar importance in staving off protein-based pathologies (Cecarini et al., 2012; de Moura et al., 2010; Green et al., 2011; Ross and Pickart, 2004). Moreover, proteasomal and mitochondrial mutants have each been identified independently as root causes of protein-aggregation and other neurodegenerative diseases (Lin and Beal, 2006). Therefore, studies of metabolic syndromes, mitochondrial disorders, and even aging-related phenomena may benefit by considering the malleable capacity of UPSdependent turnover. Apparently, proteasome dissociation/association is a reversible process that responds to cellular needs.

\section{EXPERIMENTAL PROCEDURES}

Nondenaturing PAGE and In-Gel Proteasome Activity Native protein samples were resolved by nondenaturing PAGE and proteasome activity was visualized as previously described in Glickman et al (1998a) and Bajorek et al. (2003). All gels and buffers used to assay proteasomal stability upon oxidative stress were prepared without DTT.

\section{Trichloroacetic Acid Precipitation}

Samples were prepared as previously described (Matiuhin et al., 2008; Ziv et al., 2011) and separated on an $8 \%$ SDS polyacrylamide gel to visualize ubiquitin conjugates using a ubiquitin antibody (Dako).

\section{CHX Chase}

$\mathrm{CHX}$ (Sigma) was added to logarithmic-phase cultures (after removal of oxidative reagents) to a final concentration of $200 \mu \mathrm{g} / \mathrm{ml}$. Samples were taken at the indicated time points, lysed, and loaded on native gel for activity assay. In order to detect Fzo1 levels, $\mathrm{CHX}$ chase was performed as described previously (Cohen et al., 2008, 2011).

\section{C. elegans Strains}

The Bristol N2 strain was used as the WT strain. Strains carrying reporter genes included dvls19[pAF15(Pgst-4::GFP::NLS)], hhls67 [unc-119(+); Psur-5::UbiVGFP];hhls57 [unc-119(+); sur-5::GFP]; and unc-119(ed4), zcls14[Pmyo3::GFP(mit)].

Other experimental procedures are detailed in Supplemental Experimental Procedures.

\section{SUPPLEMENTAL INFORMATION}

Supplemental Information includes Supplemental Experimental Procedures three figures, and four tables and can be found with this article online at http://dx.doi.org/10.1016/j.celrep.2014.04.030

\section{AUTHOR CONTRIBUTIONS}

N.L.-L. designed the project and executed all experiments in mammalian and yeast cells and in vitro. N.L.-L., D.K., and N.R. performed molecular biology and biochemical experiments in yeast. T.R. performed microscopy on yeast. E.K. and A.S. performed experiments with C. elegans. O.K. analyzed MS/MS data. Z.E. and M.C. analyzed Fzo1 levels. N.L.-L., T.H., and M.H.G. oversaw the project and wrote the paper. All authors read, commented on, and approved the manuscript.

\section{ACKNOWLEDGMENTS}

We thank George Lorimer (University of Maryland) for invaluable advice regarding cysteine biochemistry and crosslinking approaches, Clari Valansi and Ori Avi-Noam (Department of Biology, Technion) for assistance with cell culture and microscopy, Tamar Ziv and the dedicated staff at the Smoller Center for Proteomics (Technion) for operation of mass spectrometers and help with data generation, and Rosine Haguenauer-Tsapis and Sebastien Leon (Institute Jacques Monod) for insightful discussions. We also thank the Caenorhabditis Genetics Center (funded by the NIH Center for Research Resources), the Dana-Farber Cancer Institute, and Geneservice Ltd. for plasmids, cDNAs, and strains. This work was supported by the ATIP-Avenir Program, the "Fondation pour la Recherche Médicale" (INE 20100518343), and a Marie Curie IRG grant (276912) to M.M.C.; Deutsche Forschungsgemeinschaft grant SFB635 to T.H.; a grant from the Israel Science Foundation (ISF) to M.H.G.; and Deutsche-Israel Program DIP8 grant 2014376 to T.H. and M.H.G. 
Received: December 15, 2013

Revised: March 23, 2014

Accepted: April 16, 2014

Published: May 22, 2014

\section{REFERENCES}

Altmann, K., and Westermann, B. (2005). Role of essential genes in mitochondrial morphogenesis in Saccharomyces cerevisiae. Mol. Biol. Cell 16, 54105417.

Ambrosio, G., Zweier, J.L., Duilio, C., Kuppusamy, P., Santoro, G., Elia, P.P., Tritto, I., Cirillo, P., Condorelli, M., Chiariello, M., et al. (1993). Evidence that mitochondrial respiration is a source of potentially toxic oxygen free radicals in intact rabbit hearts subjected to ischemia and reflow. J. Biol. Chem. 268, 18532-18541.

Bader, N., and Grune, T. (2006). Protein oxidation and proteolysis. Biol. Chem. 387, 1351-1355.

Bajorek, M., Finley, D., and Glickman, M.H. (2003). Proteasome disassembly and downregulation is correlated with viability during stationary phase. Curr. Biol. 13, 1140-1144.

Bardag-Gorce, F., Oliva, J., Lin, A., Li, J., French, B.A., and French, S.W. (2011). Proteasome inhibitor up regulates liver antioxidative enzymes in rat model of alcoholic liver disease. Exp. Mol. Pathol. 90, 123-130.

Beck, F., Unverdorben, P., Bohn, S., Schweitzer, A., Pfeifer, G., Sakata, E., Nickell, S., Plitzko, J.M., Villa, E., Baumeister, W., and Förster, F. (2012). Near-atomic resolution structural model of the yeast $26 \mathrm{~S}$ proteasome. Proc. Natl. Acad. Sci. USA 109, 14870-14875.

Cecarini, V., Bonfili, L., Cuccioloni, M., Mozzicafreddo, M., Rossi, G., Buizza, L., Uberti, D., Angeletti, M., and Eleuteri, A.M. (2012). Crosstalk between the ubiquitin-proteasome system and autophagy in a human cellular model of Alzheimer's disease. Biochim. Biophys. Acta 1822, 1741-1751.

Chen, Q., Vazquez, E.J., Moghaddas, S., Hoppel, C.L., and Lesnefsky, E.J. (2003). Production of reactive oxygen species by mitochondria: central role of complex III. J. Biol. Chem. 278, 36027-36031.

Cohen, M.M., Leboucher, G.P., Livnat-Levanon, N., Glickman, M.H., and Weissman, A.M. (2008). Ubiquitin-proteasome-dependent degradation of a mitofusin, a critical regulator of mitochondrial fusion. Mol. Biol. Cell 19, 2457-2464.

Cohen, M.M., Amiott, E.A., Day, A.R., Leboucher, G.P., Pryce, E.N., Glickman, M.H., McCaffery, J.M., Shaw, J.M., and Weissman, A.M. (2011). Sequential requirements for the GTPase domain of the mitofusin Fzo1 and the ubiquitin ligase SCFMdm30 in mitochondrial outer membrane fusion. J. Cell Sci. 124, 1403-1410.

Cox, J., and Mann, M. (2008). MaxQuant enables high peptide identification rates, individualized p.p.b.-range mass accuracies and proteome-wide protein quantification. Nat. Biotechnol. 26, 1367-1372.

Davies, K.J. (2001). Degradation of oxidized proteins by the $20 \mathrm{~S}$ proteasome. Biochimie 83, 301-310.

de Moura, M.B., dos Santos, L.S., and Van Houten, B. (2010). Mitochondrial dysfunction in neurodegenerative diseases and cancer. Environ. Mol. Mutagen. 51, 391-405.

Demasi, M., Silva, G.M., and Netto, L.E. (2003). 20 S proteasome from Saccharomyces cerevisiae is responsive to redox modifications and is S-glutathionylated. J. Biol. Chem. 278, 679-685.

Finley, D. (2009). Recognition and processing of ubiquitin-protein conjugates by the proteasome. Annu. Rev. Biochem. 78, 477-513.

Friguet, B., Bulteau, A.L., and Petropoulos, I. (2008). Mitochondrial protein quality control: implications in ageing. Biotechnol. J. 3, 757-764.

Glickman, M.H., and Ciechanover, A. (2002). The ubiquitin-proteasome proteolytic pathway: destruction for the sake of construction. Physiol. Rev. 82, 373-428.

Glickman, M.H., Rubin, D.M., Coux, O., Wefes, I., Pfeifer, G., Cjeka, Z., Baumeister, W., Fried, V.A., and Finley, D. (1998a). A subcomplex of the proteasome regulatory particle required for ubiquitin-conjugate degradation and related to the COP9-signalosome and elF3. Cell 94, 615-623.

Glickman, M.H., Rubin, D.M., Fried, V.A., and Finley, D. (1998b). The regulatory particle of the Saccharomyces cerevisiae proteasome. Mol. Cell. Biol. 18, 3149-3162.

Green, D.R., Galluzzi, L., and Kroemer, G. (2011). Mitochondria and the autophagy-inflammation-cell death axis in organismal aging. Science 333, 11091112.

Grune, T., Merker, K., Sandig, G., and Davies, K.J. (2003). Selective degradation of oxidatively modified protein substrates by the proteasome. Biochem. Biophys. Res. Commun. 305, 709-718.

Hayes, J.D., Flanagan, J.U., and Jowsey, I.R. (2005). Glutathione transferases. Annu. Rev. Pharmacol. Toxicol. 45, 51-88.

Heo, J.M., Livnat-Levanon, N., Taylor, E.B., Jones, K.T., Dephoure, N., Ring, J., Xie, J., Brodsky, J.L., Madeo, F., Gygi, S.P., et al. (2010). A stress-responsive system for mitochondrial protein degradation. Mol. Cell 40, 465-480.

Kosower, N.S., and Kosower, E.M. (1995). Diamide: an oxidant probe for thiols. Methods Enzymol. 251, 123-133.

Kruegel, U., Robison, B., Dange, T., Kahlert, G., Delaney, J.R., Kotireddy, S., Tsuchiya, M., Tsuchiyama, S., Murakami, C.J., Schleit, J., et al. (2011). Elevated proteasome capacity extends replicative lifespan in Saccharomyces cerevisiae. PLoS Genet. 7, e1002253.

Layfield, R., Alban, A., Mayer, R.J., and Lowe, J. (2001). The ubiquitin protein catabolic disorders. Neuropathol. Appl. Neurobiol. 27, 171-179.

Lin, M.T., and Beal, M.F. (2006). Mitochondrial dysfunction and oxidative stress in neurodegenerative diseases. Nature 443, 787-795.

Livnat-Levanon, N., and Glickman, M.H. (2011). Ubiquitin-proteasome system and mitochondria - reciprocity. Biochim. Biophys. Acta 1809, 80-87.

Manalo, D.J., Lin, Z., and Liu, A.Y. (2002). Redox-dependent regulation of the conformation and function of human heat shock factor 1 . Biochemistry 41 , 2580-2588.

Matiuhin, Y., Kirkpatrick, D.S., Ziv, I., Kim, W., Dakshinamurthy, A., Kleifeld, O., Gygi, S.P., Reis, N., and Glickman, M.H. (2008). Extraproteasomal Rpn10 restricts access of the polyubiquitin-binding protein Dsk2 to proteasome. Mol. Cell 32, 415-425.

Merz, S., and Westermann, B. (2009). Genome-wide deletion mutant analysis reveals genes required for respiratory growth, mitochondrial genome maintenance and mitochondrial protein synthesis in Saccharomyces cerevisiae. Genome Biol. 10, R95.

Mukhopadhyay, D., and Riezman, H. (2007). Proteasome-independent functions of ubiquitin in endocytosis and signaling. Science 315, 201-205.

Okamoto, K., and Shaw, J.M. (2005). Mitochondrial morphology and dynamics in yeast and multicellular eukaryotes. Annu. Rev. Genet. 39, 503-536.

Orlowski, M., and Wilk, S. (2003). Ubiquitin-independent proteolytic functions of the proteasome. Arch. Biochem. Biophys. 415, 1-5.

Page, M.M., Robb, E.L., Salway, K.D., and Stuart, J.A. (2010). Mitochondrial redox metabolism: aging, longevity and dietary effects. Mech. Ageing Dev. 131, 242-252.

Rambold, A.S., Kostelecky, B., and Lippincott-Schwartz, J. (2011). Together we are stronger: fusion protects mitochondria from autophagosomal degradation. Autophagy 7, 1568-1569.

Reinheckel, T., Sitte, N., Ullrich, O., Kuckelkorn, U., Davies, K.J., and Grune, T. (1998). Comparative resistance of the $20 \mathrm{~S}$ and $26 \mathrm{~S}$ proteasome to oxidative stress. Biochem. J. 335, 637-642.

Rinaldi, T., Ricordy, R., Bolotin-Fukuhara, M., and Frontali, L. (2002). Mitochondrial effects of the pleiotropic proteasomal mutation mpr1/rpn11: uncoupling from cell cycle defects in extragenic revertants. Gene 286, 43-51.

Ross, C.A., and Pickart, C.M. (2004). The ubiquitin-proteasome pathway in Parkinson's disease and other neurodegenerative diseases. Trends Cell Biol. $14,703-711$. 
Rubin, D.M., Glickman, M.H., Larsen, C.N., Dhruvakumar, S., and Finley, D. (1998). Active site mutants in the six regulatory particle ATPases reveal multiple roles for ATP in the proteasome. EMBO J. 17, 4909-4919.

Scandalios, J.G. (2005). Oxidative stress: molecular perception and transduction of signals triggering antioxidant gene defenses. Braz. J. Med. Biol. Res. 38, 995-1014.

Sedensky, M.M., and Morgan, P.G. (2006). Mitochondrial respiration and reactive oxygen species in mitochondrial aging mutants. Exp. Gerontol. 41, 237-245.

Segref, A., Torres, S., and Hoppe, T. (2011). A screenable in vivo assay to study proteostasis networks in Caenorhabditis elegans. Genetics 187, 1235-1240.

Segref, A., Kevei, É., Pokrzywa, W., Schmeisser, K., Mansfeld, J., Livnat-Levanon, N., Ensenauer, R., Glickman, M.H., Ristow, M., and Hoppe, T. (2014). Pathogenesis of human mitochondrial diseases is modulated by reduced activity of the ubiquitin/proteasome system. Cell Metab. 19, 642-652.

Sheridan, C., and Martin, S.J. (2010). Mitochondrial fission/fusion dynamics and apoptosis. Mitochondrion 10, 640-648.

Shringarpure, R., Grune, T., and Davies, K.J. (2001). Protein oxidation and $20 \mathrm{~S}$ proteasome-dependent proteolysis in mammalian cells. Cell. Mol. Life Sci. 58, 1442-1450.

Shringarpure, R., Grune, T., Mehlhase, J., and Davies, K.J. (2003). Ubiquitin conjugation is not required for the degradation of oxidized proteins by proteasome. J. Biol. Chem. 278, 311-318.

Silva, G.M., Netto, L.E., Simões, V., Santos, L.F., Gozzo, F.C., Demasi, M.A., Oliveira, C.L., Bicev, R.N., Klitzke, C.F., Sogayar, M.C., and Demasi, M.
(2012). Redox control of 20 S proteasome gating. Antioxid. Redox Signal. 16 1183-1194.

Sitte, N., Merker, K., and Grune, T. (1998). Proteasome-dependent degradation of oxidized proteins in MRC-5 fibroblasts. FEBS Lett. 440, 399-402.

Taylor, E.B., and Rutter, J. (2011). Mitochondrial quality control by the ubiquitin-proteasome system. Biochem. Soc. Trans. 39, 1509-1513.

Tonoki, A., Kuranaga, E., Tomioka, T., Hamazaki, J., Murata, S., Tanaka, K., and Miura, M. (2009). Genetic evidence linking age-dependent attenuation of the $26 \mathrm{~S}$ proteasome with the aging process. Mol. Cell. Biol. 29, 1095-1106.

Tsvetkov, P., Myers, N., Moscovitz, O., Sharon, M., Prilusky, J., and Shaul, Y. (2012). Thermo-resistant intrinsically disordered proteins are efficient $20 \mathrm{~S}$ proteasome substrates. Mol. Biosyst. 8, 368-373.

Turrens, J.F. (1997). Superoxide production by the mitochondrial respiratory chain. Biosci. Rep. 17, 3-8.

Vendelbo, M.H., and Nair, K.S. (2011). Mitochondrial longevity pathways. Biochim. Biophys. Acta 1813, 634-644.

Wang, X., Yen, J., Kaiser, P., and Huang, L. (2010). Regulation of the 26S proteasome complex during oxidative stress. Sci. Signal. 3, ra88.

Yao, T.P. (2010). The role of ubiquitin in autophagy-dependent protein aggregate processing. Genes Cancer 1, 779-786.

Ziv, I., Matiuhin, Y., Kirkpatrick, D.S., Erpapazoglou, Z., Leon, S., Pantazopoulou, M., Kim, W., Gygi, S.P., Haguenauer-Tsapis, R., Reis, N., et al. (2011). A perturbed ubiquitin landscape distinguishes between ubiquitin in trafficking and in proteolysis. Mol. Cell Proteomics 10, M111.009753. 